\title{
Insulin resistance drives hepatic de novo lipogenesis in nonalcoholic fatty liver disease
}

\author{
Gordon I. Smith, ${ }^{1}$ Mahalakshmi Shankaran, ${ }^{2}$ Mihoko Yoshino, ${ }^{1}$ George G. Schweitzer, ${ }^{1}$ Maria Chondronikola, ${ }^{1}$ Joseph W. Beals, ${ }^{1}$ \\ Adewole L. Okunade, ${ }^{1}$ Bruce W. Patterson, ${ }^{1}$ Edna Nyangau, ${ }^{2}$ Tyler Field, ${ }^{2}$ Claude B. Sirlin, ${ }^{3}$ Saswata Talukdar, ${ }^{4}$ \\ Marc K. Hellerstein, ${ }^{2}$ and Samuel Klein ${ }^{1}$ \\ ${ }^{1}$ Atkins Center of Excellence in Obesity Medicine, Center for Human Nutrition, John T. Milliken Department of Medicine, Washington University School of Medicine, St. Louis, Missouri, USA. ${ }^{2}$ Department of \\ Nutritional Sciences and Toxicology, College of Natural Resources, University of California at Berkeley, Berkeley, California, USA. ${ }^{3}$ Liver Imaging Group, University of California, San Diego, La Jolla, California, \\ USA. ${ }^{4}$ Merck \& Co., San Francisco, California, USA.
}

BACKCROUND. An increase in intrahepatic triglyceride (IHTC) is the hallmark feature of nonalcoholic fatty liver disease (NAFLD) and is decreased by weight loss. Hepatic de novo lipogenesis (DNL) contributes to steatosis in individuals with NAFLD. The physiological factors that stimulate hepatic DNL and the effect of weight loss on hepatic DNL are not clear.

METHODS. Hepatic DNL, 24-hour integrated plasma insulin and glucose concentrations, and both liver and whole-body insulin sensitivity were determined in individuals who were lean $(n=14)$, obese with normal IHTC content $(n=26)$, or obese with NAFLD ( $n=27)$. Hepatic DNL was assessed using the deuterated water method corrected for the potential confounding contribution of adipose tissue DNL. Liver and whole-body insulin sensitivity was assessed using the hyperinsulinemiceuglycemic clamp procedure in conjunction with glucose tracer infusion. Six subjects in the obese-NAFLD group were also evaluated before and after a diet-induced weight loss of $10 \%$.

RESULTS. The contribution of hepatic DNL to IHTG-palmitate was $11 \%, 19 \%$, and $38 \%$ in the lean, obese, and obese-NAFLD groups, respectively. Hepatic DNL was inversely correlated with hepatic and whole-body insulin sensitivity, but directly correlated with 24-hour plasma glucose and insulin concentrations. Weight loss decreased IHTC content, in conjunction with a decrease in hepatic DNL and 24-hour plasma glucose and insulin concentrations.

CONCLUSIONS. These data suggest hepatic DNL is an important regulator of IHTC content and that increases in circulating glucose and insulin stimulate hepatic DNL in individuals with NAFLD. Weight loss decreased IHTC content, at least in part, by decreasing hepatic DNL.

TRIAL REGISTRATION. ClinicalTrials.gov NCT02706262.

FUNDING. This study was supported by NIH grants DK56341 (Nutrition Obesity Research Center), DK20579 (Diabetes Research Center), DK52574 (Digestive Disease Research Center), and RR024992 (Clinical and Translational Science Award), and by grants from the Academy of Nutrition and Dietetics Foundation, the College of Natural Resources of UCB, and the Pershing Square Foundation.

\section{Introduction}

Nonalcoholic fatty liver disease (NAFLD) is a common complication of obesity and is associated with multiorgan insulin resistance $(1,2)$, dyslipidemia (high plasma triglyceride [TG] and low HDL cholesterol concentrations) $(3,4)$, and an increased risk of diabetes and coronary heart disease $(5,6)$. The hallmark feature

Conflict of interest: SK receives research funding from Merck Sharp \& Dohme Corp., a subsidiary of Merck \& Co., and Janssen Pharmaceuticals, and has served as a consultant for Pfizer, Novo Nordisk, and Merck Sharp \& Dohme Corp. MKH receives research funding from Gilead Sciences, Pfizer, and Synergenics. ST is an employee and shareholder of Merck \& Co. Copyright: @ 2020, American Society for Clinical Investigation.

Submitted: October 10, 2019; Accepted: November 20, 2019; Published: February 4, 2020. Reference information: J Clin Invest. 2020;130(3):1453-1460.

https://doi.org/10.1172/JCl134165. of NAFLD is an increase in intrahepatic TG (IHTG) content, which accumulates when the rate of hepatic TG production is greater than the combined rates of TG export in VLDL particles and intrahepatic oxidation of TG-derived fatty acids. Data from a series of studies have shown that the rate of VLDL-TG secretion is increased $(2,7,8)$ and that hepatic fatty acid oxidation is likely normal or increased in individuals with NAFLD (9-11), demonstrating that an increase in IHTG production rather than a decrease in IHTG mobilization is the primary mechanism for developing and maintaining hepatic steatosis. Fatty acids used for IHTG production are derived from (a) fatty acids released into the systemic and portal circulations by lipolysis of TGs in subcutaneous and intra-abdominal adipose tissues (IAATs); (b) fatty acids released into the systemic circulation by postprandial lipolysis of TGs in chylomicrons; (c) hepatic lipolysis of TGs 
Table 1. Body composition and metabolic characteristics of the study subjects

\begin{tabular}{|c|c|c|c|}
\hline & Lean $(n=14)$ & Obese $(n=26)$ & Obese-NAFLD $(n=27)$ \\
\hline $\mathrm{BMI}\left(\mathrm{kg} / \mathrm{m}^{2}\right)$ & $22.6 \pm 0.4$ & $37.0 \pm 0.9^{A}$ & $38.9 \pm 0.9^{A}$ \\
\hline Body fat (\%) & $29.4 \pm 1.5$ & $48.0 \pm 1.2^{\mathrm{A}}$ & $47.7 \pm 1.1^{\mathrm{A}}$ \\
\hline IHTG content (\%) & $1.8 \pm 0.2$ & $2.3 \pm 0.2$ & $18.0 \pm 1.7^{A, B}$ \\
\hline IAAT volume $\left(\mathrm{cm}^{3}\right)$ & $400 \pm 55$ & $917 \pm 71^{\AA}$ & $1864 \pm 130^{A, B}$ \\
\hline ASAT volume $\left(\mathrm{cm}^{3}\right)$ & $937 \pm 105$ & $3716 \pm 215^{A}$ & $3644 \pm 240^{A}$ \\
\hline HbA1c (\%) & $5.0 \pm 0.1$ & $5.0 \pm 0.1$ & $5.7 \pm 0.1^{A, B}$ \\
\hline $\mathrm{TCs}$ (mg/dL) & $67 \pm 8$ & $67 \pm 4$ & $141 \pm 13^{A, B}$ \\
\hline HDL cholesterol (mg/dL) & $67 \pm 4$ & $55 \pm 3^{A}$ & $43 \pm 2^{A, B}$ \\
\hline LDL cholesterol (mg/dL) & $99 \pm 6$ & $99 \pm 5$ & $118 \pm 6$ \\
\hline Fasting glucose (mg/dL) & $85 \pm 1$ & $88 \pm 1$ & $101 \pm 2^{A, B}$ \\
\hline Clucose: 2 hours OGTT (mg/dL) & $96 \pm 5$ & $106 \pm 3$ & $170 \pm 6^{A, B}$ \\
\hline Glucose: 24 hours AUC (mg/dL $\times 24$ hours) & $2260 \pm 46$ & $2302 \pm 27$ & $2732 \pm 56^{A, B}$ \\
\hline Fasting insulin $(\mu \mathrm{U} / \mathrm{mL})$ & $5.2 \pm 0.5$ & $11.8 \pm 1.4$ & $27.1 \pm 3.4^{A, B}$ \\
\hline Insulin: 24 hours AUC ( $\mu \mathrm{U} / \mathrm{mL} \times 24$ hours) & $564 \pm 81$ & $1059 \pm 89$ & $2168 \pm 252^{A, B}$ \\
\hline HISI & $10.8 \pm 1.0$ & $5.8 \pm 0.4^{\mathrm{A}}$ & $3.0 \pm 0.2^{\mathrm{A}, \mathrm{B}}$ \\
\hline Clucose Rd during the HECP ( $\mu \mathrm{mol} / \mathrm{kg}$ FFM/min) & $60.8 \pm 3.5$ & $48.3 \pm 2.4^{A}$ & $27.6 \pm 1.4^{A, B}$ \\
\hline
\end{tabular}

Data are expressed as the mean \pm SEM. One-way ANOVA was performed to compare subject characteristics. Tukey's post hoc test was used to identify significant mean differences between groups where appropriate. ${ }^{A}$ Value significantly different from the corresponding value in the lean group, $P<0.05$. ${ }^{B}$ Value significantly different from the corresponding value in the obese group, $P<$ 0.05. ASAT, abdominal subcutaneous adipose tissue.
Although the mechanism(s) responsible for the increase in hepatic DNL in individuals with NAFLD is not known, it is possible that increases in circulating insulin and glucose associated with hepatic and whole-body insulin resistance are involved in the stimulation of hepatic DNL, because insulin and glucose activate sterol regulatory element-binding protein 1c (SREBP-1c) and carbohydrate response elementbinding protein (ChREBP), respectively (25-30), which transcriptionally activate genes involved in DNL. This mechanism implies differential effects of insulin action on specific metabolic functions in the liver, manifested by insulin resistance with respect to the suppression of hepatic glucose production, but preserved insulin sensitivity with respect to the SREBP-1c pathway that stimulates fatty acid synthesis. Studies conducted in primary hepatocytes and in rodent models of obesity and diabetes support the notion of selective pathway-specific insulin resistance in the liver (31-34).

in plasma lipoproteins delivered to the liver; and (d) fatty acids synthesized de novo from nonlipid precursors in the liver $(12,13)$.

The de novo synthesis of fatty acids in the liver involves a complex cytosolic polymerization process in which acetyl-CoA is converted to malonyl-CoA, which then undergoes several cycles of condensation, decarboxylation, and reduction reactions to form 1 palmitate molecule. De novo lipogenesis (DNL) in the liver can be measured in vivo by using isotopically labeled tracers to assess the synthesis rate of palmitate secreted in liver-derived TGs into plasma. Studies using this approach reported that DNL accounts for $15 \%$ to $26 \%$ of IHTG-palmitate production in individuals with NAFLD $(12,14-16)$ and $1 \%$ to $10 \%$ of IHTG-palmitate production in individuals who are either lean or obese with normal IHTG content $(14,17-20)$. It is possible, however, that these studies underestimated the contribution from DNL because (a) most studies were conducted when participants were in the fasted state when DNL is lowest $(19,21)$; (b) the duration of isotope administration was not long enough to detect newly synthesized fatty acids that were incorporated into and released from slowly turning-over IHTG pools $(22,23)$, a concept supported by the results of a recent clinical trial in which deuterated water $\left(\mathrm{D}_{2} \mathrm{O}\right)$ was administered for a more prolonged period and showed a greater contribution of DNL to IHTG production in subjects with nonalcoholic steatohepatitis than reported previously (24); and (c) the correction factor used to account for the contribution of fatty acids made by DNL in adipose tissue (i.e., fatty acids made de novo in adipose tissue that were released into the bloodstream and subsequently incorporated into VLDL-TG in the liver) was too high, because it was assumed that all plasma free fatty acids (FFAs) made by DNL were derived from adipose tissue without any contribution of plasma FFAs from lipolysis of circulating TGs produced by the liver (14).
The purpose of the present study was to (a) determine hepatic DNL, measured over a prolonged period ( $3-5$ weeks) of daily $\mathrm{D}_{2} \mathrm{O}$ ingestion and corrected for the contribution of fatty acids made de novo in adipose tissue, in 3 distinct cohorts of individuals who were lean with normal oral glucose tolerance and normal IHTG content (lean), obese with normal oral glucose tolerance and normal IHTG content (obese), or obese with abnormal oral glucose tolerance and NAFLD (obese-NAFLD); (b) determine the relationships among hepatic DNL and IHTG content and key factors that are probably involved in regulating DNL, namely liver and whole-body insulin sensitivity and integrated 24-hour plasma insulin and glucose concentrations; and (c) determine the effect of moderate (10\%) weight loss on hepatic DNL, IHTG content, liver and whole-body insulin sensitivity, and integrated 24-hour plasma insulin and glucose concentrations. We assessed liver and whole-body insulin sensitivity using the hyperinsulinemic-euglycemic clamp procedure (HECP) in conjunction with stable isotopically labeled glucose tracer infusion, and we assessed a 24-hour integration of plasma insulin and glucose concentrations by serial blood sampling. We hypothesized that hepatic DNL would negatively correlate with hepatic and whole-body insulin sensitivity and positively correlate with 24-hour plasma glucose and insulin concentrations and IHTG content and that moderate weight loss would decrease hepatic DNL in concert with improvements in insulin sensitivity and a decrease in the integrated 24-hour plasma insulin and glucose concentrations.

\section{Results}

Body composition and metabolic characteristics. The obese and obese-NAFLD groups were matched in terms of percentage of body fat, but IAAT volume and IHTG content were much great- 

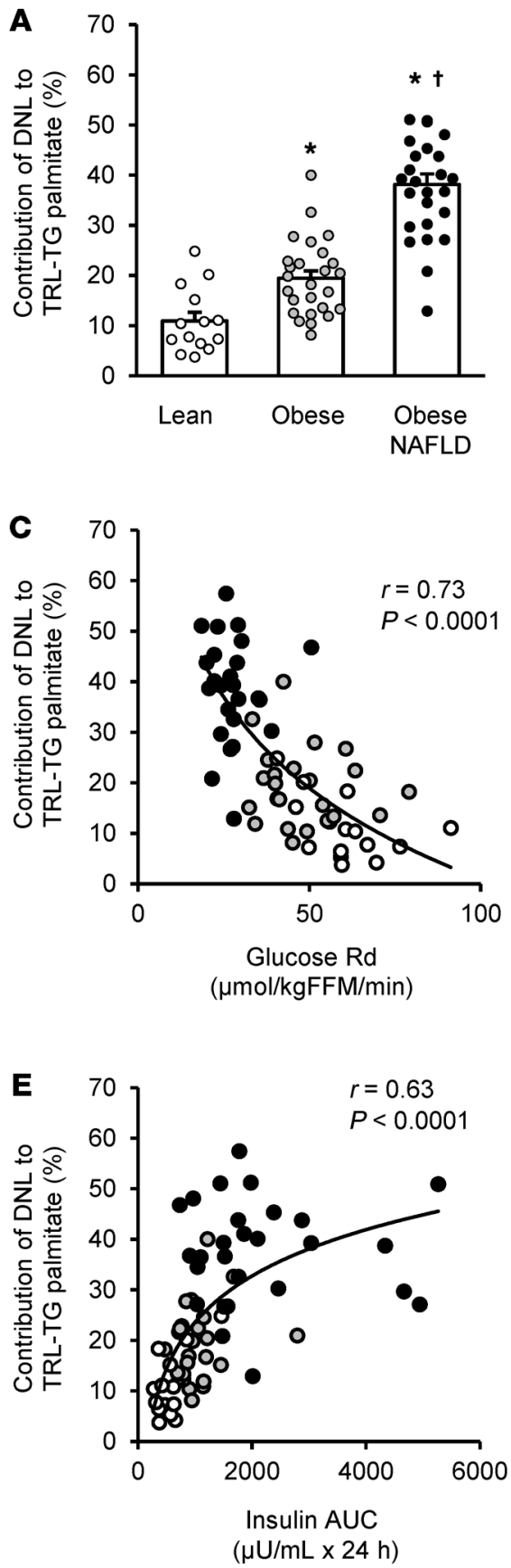
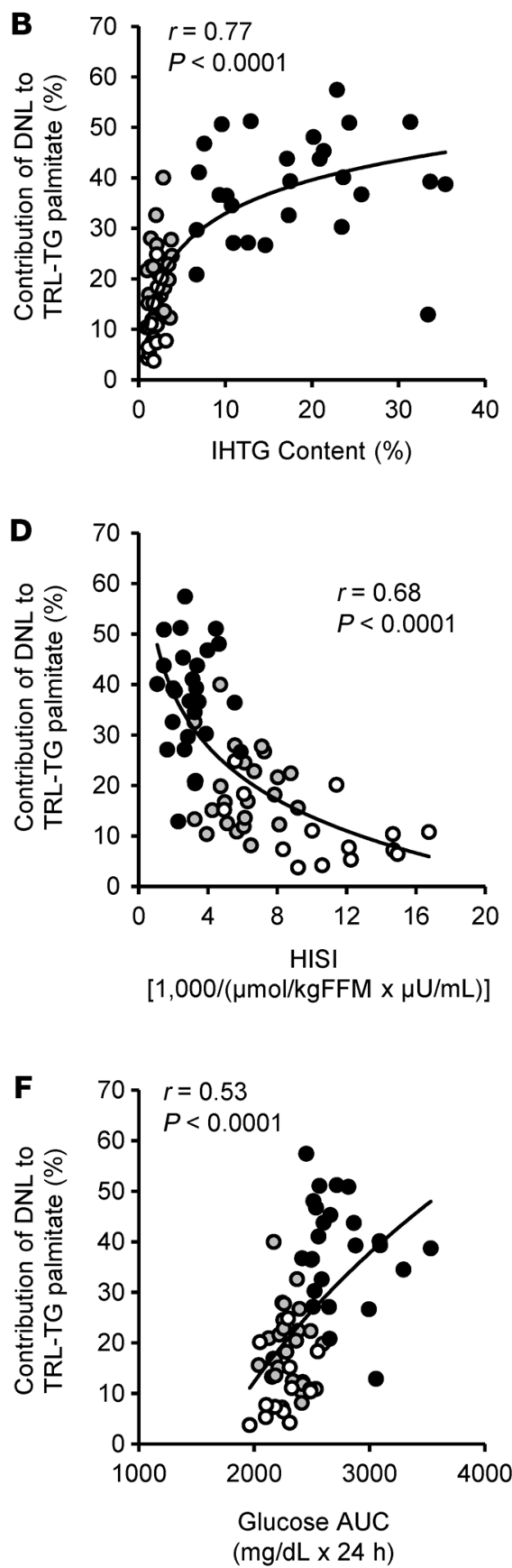

Figure 1. Relationships among hepatic DNL and metabolic characteristics. (A) Relative contribution of DNL to IHTC content, assessed as palmitate produced by DNL, measured in plasma TG-rich lipoprotein TGs (TRL-TGs) in 3 groups of subjects: lean with normal IHTC content (Lean; $n=14$ ); obese with normal IHTC content (Obese; $n=$ 26); and obese with NAFLD (Obese-NAFLD; $n=27)$. Values indicate the mean \pm SEM. One-way ANOVA was performed to compare the relative contribution of DNL to TRL-TC palmitate, with Tukey's post hoc test used to identify significant mean differences between groups. *Value significantly different from the lean group value, $P<0.05$. 'Value significantly different from the obese group value, $P<0.01$. Relationships between hepatic DNL, assessed as the percentage of contribution of DNL to plasma TRL-TC palmitate and (B) IHTC content; (C) whole-body insulin sensitivity, assessed as the glucose Rd during a HECP; (D) HISI; and integrated 24-hour AUCs for plasma (E) insulin and (F) glucose. Logarithmic regression analysis was used to determine the lines of best fit to the data. White, gray, and black circles represent participants in the lean, obese, and obese-NAFLD groups, respectively. er in the obese-NAFLD group than in the obese group (Table 1). In addition, fasting plasma TGs, glucose and insulin concentrations, hemoglobin A1c (HbA1c), 2-hour oral glucose tolerance test (OGTT) plasma glucose, and the integrated 24-hour plasma glucose and insulin AUC values were greater, and hepatic and wholebody insulin sensitivity were lower, in the obese-NAFLD group than in the obese group. Although many metabolic variables in the obese group did not differ from those in the lean group, hepatic and whole-body insulin sensitivity was lower in the obese group.

Hepatic DNL progressively increases with increases in insulin-resistant glucose metabolism. Total body water enrichment with deuterium after prolonged daily $\mathrm{D}_{2} \mathrm{O}$ consumption did not differ between groups and was $1.80 \% \pm 0.14 \%, 1.73 \% \pm 0.07 \%$, and $1.80 \% \pm$
$0.07 \%$ in the lean, obese, and obese-NAFLD groups, respectively $(P=0.81)$. DNL in adipose tissue TGs was very minimal and did not differ between groups: $1.4 \% \pm 0.1 \%$ of palmitate in adipose TGs was derived from DNL, which represents the combined average of $1.7 \% \pm 0.5 \%, 1.2 \% \pm 0.1 \%$, and $1.5 \% \pm 0.1 \%$ in the lean, obese, and obese-NAFLD groups, respectively $(P=0.26)$. The proportion of plasma free palmitate produced by DNL $(8.8 \% \pm 0.8 \%)$ was more than 5 -fold greater than the proportion of palmitate made by DNL in adipose tissue (Supplemental Figure 1; supplemental material available online with this article; https://doi.org/10.1172/ JCI134165DS1). Accordingly, more than $80 \%$ of the labeled plasma free palmitate could not have come from adipose tissue fatty acids and must have come from the DNL pathway in liver and been 
A

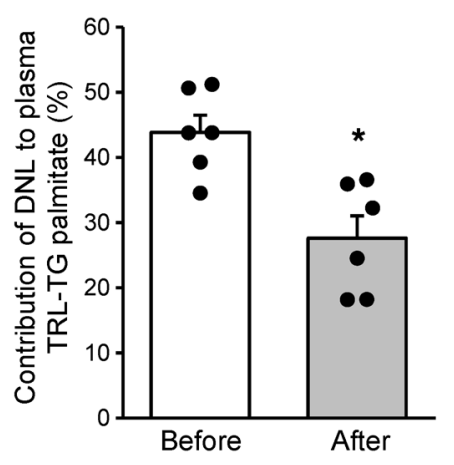

B

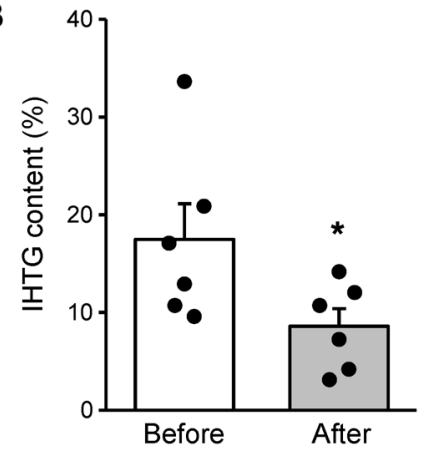

Figure 2. Moderate weight loss decreases hepatic DNL and IHTC content in subjects with obesity and NAFLD. Relative contribution of DNL to IHTC content, assessed as palmitate produced by DNL within plasma TRL-TC palmitate (A) and IHTC content (B) before and after a weight loss of approximately $10 \%$ in 6 individuals with obesity and NAFLD. Values indicate the mean \pm SEM. Circles represent individual values before and after weight loss. Student's $t$ test for paired samples was used to assess the statistical significance of differences in values before and after weight loss. Asterisks indicate the value significantly different from the before value, $P<0.05$. subsequently released into the bloodstream by lipolysis of TGs in circulating liver-derived TG-rich lipoproteins (TRLs) (35). The relative contribution of hepatic DNL to total IHTG-palmitate synthesis (calculated from the palmitate made by DNL in circulating TRL-TGs, after subtracting the contribution from palmitate made by DNL in adipose tissue) was lowest in the lean group $(10.9 \% \pm$ $1.7 \%)$ and nearly 2 -fold $(19.4 \% \pm 1.5 \%)$ and 3.5 -fold $(38.5 \% \pm 2.0 \%)$ higher in the obese and obese-NAFLD groups, respectively (Figure 1A). The relative contribution of DNL to plasma TRL-TG palmitate positively correlated with IHTG content (Figure 1B), negatively correlated with both whole-body and hepatic insulin sensitivity (Figure 1, C and D), and positively correlated with 24-hour plasma insulin and glucose AUC values (Figure 1, E and F).

Moderate weight loss causes a marked decrease in hepatic DNL and IHTG content. Six subjects in the obese-NAFLD group repeated the testing procedures after a diet-induced weight loss of $10.3 \% \pm 0.8 \%$ (range 7.3\%-12.1\%). Body weight was stable throughout the periods of $\mathrm{D}_{2} \mathrm{O}$ consumption before and after weight loss $(0.8 \% \pm 0.2 \%$ change in weight during both periods of $\mathrm{D}_{2} \mathrm{O}$ administration), and total body water deuterium enrichment after weight loss did not differ from the values before weight loss $(1.69 \% \pm 0.23 \%$ and $1.78 \% \pm$ $0.14 \%$, respectively; $P=0.57$ ). Weight loss decreased the relative contribution of hepatic DNL to total IHTG-palmitate synthesis by $35 \% \pm$ $10 \%$ and IHTG content by $50 \% \pm 8 \%$ (Figure 2 , A and B). Weight loss also improved hepatic and whole-body insulin sensitivity, decreased 24-hour plasma glucose and insulin AUCs, and decreased HbA1c, plasma TG, and LDL cholesterol concentrations (Table 2).

\section{Discussion}

Steatosis is the hallmark feature of NAFLD and precedes the progression to steatohepatitis and fibrosis (36). Accordingly, an understanding of the mechanisms responsible for the excessive accumulation of IHTG has important physiological and clinical implications. Using a prolonged oral $\mathrm{D}_{2} \mathrm{O}$ administration approach to assess hepatic DNL and correcting for the contribution of fatty acids made by DNL in adipose tissue TGs, we found a much larger contribution of DNL to IHTG formation in individuals with obesity and NAFLD than previously appreciated; on average, nearly $40 \%$ of plasma TRL-TG palmitate was derived from hepatic DNL, and the relative contribution of DNL was directly correlated with IHTG content. We also found that individuals with obesity and normal IHTG content had a much greater contribution from hepatic DNL to IHTG formation than did lean individuals. In addition, our data demonstrate a strong negative correlation between the rate of DNL and both whole-body and hepatic insulin sensitivity and positive correlations with integrated 24-hour plasma glucose and insulin concentrations. An increase in hepatic and whole-body insulin sensitivity and a decrease in 24-hour plasma glucose and insulin concentrations induced by moderate (10\%) weight loss were also associated with a marked decrease in both hepatic DNL and IHTG content. Taken together, these data underscore the importance of DNL in the pathogenesis of hepatic steatosis and suggest that increases in daily 24 -hour plasma glucose and insulin concentrations are major drivers of increased DNL in individuals with obesity and NAFLD. Moreover, this clinical study supports the notion that the selective hepatic insulin resistance demonstrated in cell systems and animal models $(31,33,34)$ is involved in the pathogenesis of NAFLD in humans.

The values we observed for the contribution of DNL to IHTG fatty acid production in our lean, obese, and obese-NAFLD groups were greater than those reported previously in similar study populations $(12,14-17,22,37,38)$. The reason for the differences between our results and those of other studies is probably relat-
Table 2. Body composition and metabolic characteristics of the study subjects before and after a $10 \%$ weight loss

\begin{tabular}{|c|c|c|c|}
\hline & Before & After & $P$ value \\
\hline $\mathrm{BMI}\left(\mathrm{kg} / \mathrm{m}^{2}\right)$ & $37.7 \pm 1.6$ & $34.4 \pm 1.6$ & - \\
\hline Body fat $(\%)$ & $47.2 \pm 2.0$ & $43.5 \pm 2.0$ & 0.02 \\
\hline HbA1c $(\%)$ & $5.8 \pm 0.2$ & $5.4 \pm 0.2$ & 0.03 \\
\hline $\mathrm{TCs}(\mathrm{mg} / \mathrm{dL})$ & $151 \pm 11$ & $118 \pm 9$ & 0.04 \\
\hline HDL cholesterol (mg/dL) & $44 \pm 2$ & $38 \pm 1$ & $<0.01$ \\
\hline LDL cholesterol (mg/dL) & $122 \pm 6$ & $100 \pm 9$ & 0.02 \\
\hline Fasting glucose (mg/dL) & $106 \pm 6$ & $99 \pm 3$ & 0.15 \\
\hline Glucose: 24 hours AUC (mg/dL × 24 hours) & $2870 \pm 117$ & $2559 \pm 121$ & $<0.001$ \\
\hline Insulin: 24 hours $\mathrm{AUC}(\mu \mathrm{U} / \mathrm{mL} \times 24$ hours) & $2135 \pm 369$ & $1183 \pm 137$ & 0.02 \\
\hline HISI & $2.5 \pm 0.4$ & $4.9 \pm 0.9$ & 0.04 \\
\hline Glucose Rd during the HECP ( $\mu \mathrm{mol} / \mathrm{kg} \mathrm{FFM} / \mathrm{min}$ ) & $25.6 \pm 1.7$ & $40.7 \pm 2.8$ & $<0.01$ \\
\hline
\end{tabular}

Data are expressed as the mean \pm SEM. All $P$ values were calculated using Student's $t$ test for paired samples. 
ed to several factors. First, the long (3- to 5-week) duration of $\mathrm{D}_{2} \mathrm{O}$ tracer administration in our study accounted for labeling of the liver TG pools that turn over slowly (12) and provided an integrated assessment of DNL during daily fasting and fed conditions over several weeks. In contrast, the assessment of hepatic DNL in most other studies was conducted with a much shorter period of tracer administration and was conducted during fasting conditions when DNL is reduced. Second, we avoided overestimating the potential contribution of fatty acids synthesized de novo in adipose tissue that could have been released into the circulation and incorporated into VLDL-TGs in the liver (14-17). We did a direct measurement of DNL-derived palmitate in adipose tissue TGs and estimated the incorporation of flux from this source of fatty acids into VLDL-TGs in the liver. DNL accounted for less than $2 \%$ of palmitate in adipose TGs but $9 \%$ in plasma free palmitate, indicating that other sources of fatty acids, presumably spillover of fatty acids into the circulation during lipoprotein lipase-mediated lipolysis of TRL-TGs derived from the liver, must contribute to the pool of circulating DNL-derived FFAs. Finally, we evaluated DNL in precisely defined cohorts of individuals who were segregated into distinct groups on the basis of BMI, oral glucose tolerance, and IHTG content. Therefore, it is possible that differences in study populations between our study and others contributed to differences in hepatic DNL.

The mechanism or mechanisms responsible for the increase in hepatic DNL in individuals with NAFLD are not known. Our data suggest that daily 24-hour increases in plasma insulin and glucose concentrations associated with insulin-resistant glucose metabolism contribute to the stimulation of hepatic DNL. Data from studies conducted in cell systems and rodent models have shown that insulin and glucose independently regulate hepatic DNL by activating SREBP-1c and ChREBP, respectively (25, 27-30,39), which in turn activate nearly all the genes involved in hepatic DNL. Hepatic expression of SREBP-1c, ChREBP, and other genes involved in lipogenesis are increased in individuals with hepatic steatosis compared with those who have normal IHTG content (40-44). We found positive relationships between hepatic DNL and the integrated 24-hour plasma concentrations of both glucose and insulin and a negative relationship between hepatic DNL and the hepatic insulin sensitivity index (HISI). These findings support the notion of a dissociation between insulin action on hepatic glucose and lipid metabolism in individuals with NAFLD, manifested by insulin-resistant hepatic glucose metabolism but insulin-sensitive hepatic lipogenesis. In addition, it is possible that the process of DNL itself produces toxic metabolites, such as diacylglycerol and ceramides, that can induce insulin resistance $(45$, 46), thereby establishing a positive feedback loop in which insulin resistance stimulates hepatic DNL and hepatic DNL contributes to insulin resistance.

We believe our study has important implications in the development of drug therapies that modulate IHTG content. The therapeutic potential of modulating DNL to decrease IHTG content in individuals with NAFLD has been demonstrated in studies that involved novel pharmacologic agents. The data from 1 study showed that liver-specific inhibition of acetyl-CoA carboxylase, a major regulator of hepatic DNL, caused a marked decrease in hepatic DNL and IHTG content in subjects with NAFLD (47).
The influence of insulin action in the liver on IHTG content was demonstrated in a randomized, controlled trial conducted in adults with insulin-treated type 2 diabetes who were randomized to therapy with either peglispro (Eli Lilly and Co.), a polyethylene glycolylated insulin analog, or insulin glargine (48). Treatment with peglispro, which has preferential action in the liver and decreased peripheral action compared with insulin glargine (49), caused a $50 \%$ increase in IHTG content, assessed at 26 and 52 weeks of therapy, whereas treatment with insulin glargine did not affect IHTG content. Collectively, these results further underscore the important role of DNL in developing and maintaining steatosis in individuals with NAFLD.

Weight loss has profound effects on IHTG content. Even 48 hours of calorie restriction causes a marked decline in IHTG content (50), and at any given percentage of weight loss, the relative decrease in IHTG content is much greater than the relative decrease in whole-body fat mass or IAAT (51). In the present study, we found that moderate $(10 \%)$ weight loss caused a marked decrease in both hepatic DNL and IHTG content, suggesting that a decrease in hepatic DNL is an important mechanism responsible for the weight loss-induced decline in IHTG content.

In summary, we found that hepatic DNL, when assessed by a labeling protocol of sufficient duration to account for large, slowly turning-over hepatic TG pools, is an important contributor to IHTG production and IHTG content in individuals with NAFLD. Moreover, by carefully selecting participants with a wide range of insulin sensitivity and IHTG content, we were able to demonstrate that the contribution of hepatic DNL to IHTG was negatively correlated with both hepatic and whole-body insulin sensitivity and positively correlated with integrated 24 -hour plasma glucose and insulin concentrations. These data suggest that increases in circulating glucose and insulin associated with insulin resistance promote hepatic DNL in individuals with NAFLD. In contrast, an improvement in insulin sensitivity and a concomitant decrease in hepatic DNL are potentially important contributors to the decline in IHTG content associated with moderate weight loss.

\section{Methods}

Subjects. A total of 445 potential subjects were screened for this study. A total of 67 men and women (mean age: $39 \pm 1$ years; 14 men and 53 women) who were eligible and willing to participate and completed all baseline testing were included in the cross-sectional comparison of lean, obese, and obese-NAFLD subjects. The study flow chart is shown in Supplemental Figure 2. Subjects were recruited between April 2016 and November 2018 via the Volunteers for Health database at the Washington University School of Medicine and via local postings. All parts of this study were conducted in the Clinical Translational Research Unit (CTRU) and the Center for Clinical Imaging Research (CCIR) at Washington University School of Medicine. To determine eligibility, subjects underwent a comprehensive screening evaluation including a medical history and physical examination, standard blood tests, a test for HbA1c, an OGTT, and assessment of IHTG content using MRI. The following inclusion criteria were required for each cohort: (a) lean group ( $n=14,8$ women), a BMI of $18.5-24.9 \mathrm{~kg} / \mathrm{m}^{2}$, IHTG content of $4 \%$ or less, a serum TG concentration below $150 \mathrm{mg} / \mathrm{dL}$, a fasting plasma glucose concentration below $100 \mathrm{mg} / \mathrm{dL}$, a 2-hour OGTT plasma glucose concentration 
below $140 \mathrm{mg} / \mathrm{dL}$, and a HbA1c of $5.6 \%$ or less; (b) obese group ( $n$ = 26, 24 women), a BMI of 30-49.9 kg/m², IHTG content of $4 \%$ or less, a serum TG concentration below $150 \mathrm{mg} / \mathrm{dL}$, a fasting plasma glucose concentration below $100 \mathrm{mg} / \mathrm{dL}$, a 2-hour OGTT plasma glucose concentration below $140 \mathrm{mg} / \mathrm{dL}$, and a $\mathrm{HbA1c}$ of $5.6 \%$ or less; (c) obese-NAFLD group ( $n=27,21$ women), a BMI of 30-49.9 $\mathrm{kg} / \mathrm{m}^{2}$, IHTG content of $6.0 \%$ or higher, and a HbA1c of $5.7 \%-6.4 \%$, or a fasting plasma glucose concentration of $100 \mathrm{mg} / \mathrm{dL}$ or higher, or a 2-hour OGTT plasma glucose concentration of $140 \mathrm{mg} / \mathrm{dL}$ or higher. Potential participants who had a history of diabetes or liver disease other than NAFLD, were taking medications that can affect metabolism or cause liver damage, or consumed excessive amounts of alcohol (more than $21 \mathrm{oz}$ of alcohol per week for men or more than $14 \mathrm{oz}$ of alcohol per week for women) were excluded.

Body composition analyses. Body fat mass and fat-free mass (FFM) were determined by dual-energy x-ray absorptiometry (DXA) (Lunar iDXA, GE Healthcare Lunar). Abdominal subcutaneous adipose tissue and IAAT volumes and IHTG content were determined by MRI (3T superconducting magnet, Siemens) as previously described $(52,53)$.

Integrated 24-hour plasma glucose and insulin concentrations and insulin sensitivity. Subjects were admitted to the CTRU at 1700 hours for approximately 48 hours and consumed a standard meal (50\% carbohydrate, $35 \%$ fat, $15 \%$ protein) containing one-third of their estimated energy requirements (54) between 1800 hours and 1900 hours. At 0630 hours the next morning on day 2, a catheter was inserted into an antecubital vein for 24-hour serial blood sampling. Blood samples were obtained every hour from 0700 hours to 2300 hours on day 2 and from 0500 hours to 0700 hours on day 3 , and additional blood samples were obtained every 30 minutes for 2 hours after each meal. Meals were provided at 0700 hours, 1300 hours, and 1900 hours. Each meal contained one-third of the participant's energy requirements and consisted of $50 \%$ carbohydrate, $35 \%$ fat, and $15 \%$ protein. A HECP, in conjunction with stable isotopically labeled glucose tracer infusion, was conducted on day 3 to assess hepatic and whole-body insulin sensitivity. At 0700 hours, a primed $(8.0 \mu \mathrm{mol} / \mathrm{kg})$ continuous $(0.08 \mu \mathrm{mol} / \mathrm{kg} /$ $\min$ ) infusion of $\left[\mathrm{U}^{-13} \mathrm{C}\right]$ glucose (Cambridge Isotope Laboratories Inc.) was started through the existing intravenous catheter. An additional catheter was inserted into a radial artery to obtain arterial blood samples. After the infusion of glucose tracer for 210 minutes (basal period), insulin was infused for 210 minutes at a rate of $50 \mathrm{mU} / \mathrm{m}^{2}$ body surface area (BSA) $/ \mathrm{min}$ (initiated with a 2-step priming dose of $200 \mathrm{mU} / \mathrm{m}^{2} \mathrm{BSA} / \mathrm{min}$ for 5 minutes followed by $100 \mathrm{mU} / \mathrm{m}^{2} \mathrm{BSA} / \mathrm{min}$ for 5 minutes). The infusion of $\left[\mathrm{U}-{ }^{13} \mathrm{C}\right]$ glucose was stopped during insulin infusion because of the expected decrease in hepatic glucose production (55). Euglycemia (approximately $100 \mathrm{mg} / \mathrm{dL}$ ) was maintained by variable infusion of $20 \%$ dextrose enriched to approximately $1 \%$ with $\left[\mathrm{U}-{ }^{13} \mathrm{C}\right] \mathrm{glu}-$ cose. Blood samples were obtained before beginning the tracer infusion and every 6 to 7 minutes during the final 20 minutes (total of 4 blood samples) of the basal and insulin infusion periods.

DNL. Subjects consumed $50-\mathrm{mL}$ aliquots of $70 \% \mathrm{D}_{2} \mathrm{O}$ (Sigma-Aldrich), provided in sterile vials, every day for 3 to 5 weeks; aliquots of $\mathrm{D}_{2} \mathrm{O}$ were consumed 3 to 4 times/day every day for the first 5 days (priming period) followed by two $50-\mathrm{mL}$ doses daily. The final aliquot of $\mathrm{D}_{2} \mathrm{O}$ was taken on the evening of day 2 of the inpatient CTRU admission. A blood sample obtained at 0700 hours the following morning was used to determine body water $\mathrm{D}_{2} \mathrm{O}$ enrichment and hepatic DNL. Compliance with $\mathrm{D}_{2} \mathrm{O}$ consumption was monitored by interview at weekly visits with the study research coordinator, by counting the return of empty vials at each visit, and by evaluating $\mathrm{D}_{2} \mathrm{O}$ enrichments in plasma (obtained on day 7 and weekly thereafter) and saliva (obtained on days 2, 4, and 11 and then weekly thereafter). To evaluate the potential confounding contribution of fatty acids made de novo in adipose tissue to our measurement of hepatic DNL, we measured DNL of palmitate in plasma FFAs and in abdominal adipose tissue TGs. Abdominal subcutaneous adipose tissue was obtained by percutaneous biopsy during the basal stage of the HECP as previously described (2).

Diet intervention and post-weight loss testing. After baseline testing was completed, 7 subjects in the obese-NAFLD group participated in a weight loss program supervised by our study dietitian and behavioral psychologist, involving weekly individual dietary and behavioral education sessions with all food provided as prepackaged meals. The macronutrient content of the diet was composed of approximately $50 \%$ of energy as carbohydrate, approximately $30 \%$ as fat, and approximately $15 \%$ as protein. The initial daily energy content of the diet provided $75 \%$ of estimated energy requirements (54); subsequent meals and energy intake were adjusted weekly as needed to achieve a $0.5 \%-1 \%$ weight loss per week until approximately $10 \%$ weight loss was achieved, which took approximately 25 weeks. Once the targeted weight loss goal was achieved, dietary energy intake was modified to maintain a stable body weight for 3 to 4 weeks before the testing procedures performed at baseline were repeated. One subject withdrew from the study because of an inability to lose weight; data from 6 subjects are reported here.

Sample analyses. Plasma glucose concentration was determined by using an automated glucose analyzer (Yellow Spring Instruments Co.). Plasma insulin was measured using electrochemiluminescence technology (Elecsys 2010, Roche Diagnostics). Plasma TG and HDL cholesterol concentrations were determined enzymatically by colorimetric assays (Roche Diagnostics). Plasma LDL cholesterol concentration was calculated according to the Friedewald formula (56). HbA1c was measured by turbidimetric inhibition immunoassay (Roche Diagnostics). Deuterium enrichment in total body water, deuterium enrichment and labeling pattern in plasma FFAs, TRL-TGs, and adipose tissue TGs, and $\left[\mathrm{U}-{ }^{13} \mathrm{C}\right.$ ]glucose enrichment in plasma glucose were determined by using gas chromatography-mass spectrometry (GC-MS) as described previously $(57,58)$.

Calculations. Plasma glucose and insulin AUC over a 24-hour period were calculated using the trapezoidal method (59). The HISI was calculated as the inverse of the product of plasma insulin concentration and the endogenous glucose rate of appearance (Ra) in the systemic circulation, determined by dividing the glucose tracer infusion rate by the average plasma glucose tracer-to-tracee ratio (TTR) during the last 20 minutes of the basal period of the HECP (1). The total glucose rate of disappearance (Rd) during insulin infusion was assumed to be equal to the sum of the endogenous glucose $\mathrm{Ra}$ and the rate of infused glucose during the last 20 minutes of the HECP (1). The fractional contribution of DNL to palmitate in plasma FFAs, plasma TRL-TGs, and adipose tissue TGs was calculated by mass isotopomer distribution analy- 
sis as described previously $(17,58)$. Circulating TRL-TG palmitate can be used to assess hepatic DNL, because the fatty acid composition and the source of fatty acids in IHTGs and in circulating TRL-TGs are the same (12). Accordingly, hepatic DNL was calculated as the measured total contribution of palmitate made by DNL in circulating TRL-TGs minus the estimated contribution of palmitate made by DNL in adipose tissue TGs that were released into the circulation and incorporated into TRL-TGs in the liver. The contribution of palmitate synthesized de novo in adipose tissue and incorporated into circulating TRL-TGs was estimated by (a) direct measurement of the contribution of palmitate made by DNL in adipose tissue TGs; and (b) an estimation of the relative incorporation into the circulation of these released fatty acids that were then incorporated into VLDL-TGs in the liver. This estimation was based on the results of our previous studies showing that the contribution of systemic plasma FFAs to VLDL-TGs secreted by the liver was $75 \%, 65 \%$, and $40 \%$ in similar groups of subjects who were lean, obese with normal IHTG content, and obese with NAFLD, respectively $(2,7,60)$.

Statistics. One-way ANOVA was performed to compare characteristics of the study participants and outcome measures between lean, obese, and obese-NAFLD groups, using Tukey's post hoc test to locate significant mean differences where appropriate. Student's $t$ test for paired samples was used to assess the statistical significance of differences in values before and after weight loss. Relationships among DNL, IHTG content, and selected metabolic variables were evaluated by linear and nonlinear regression analysis. In all instances, a logarithmic regression curve provided the best fit to the data. All statistical tests were 2-sided, with a $P$ value of less than 0.05 considered statistically significant. Data are presented as the mean \pm SEM unless otherwise noted. Statistical analyses were performed using SPSS software (version 25, IBM).

On the basis of the interindividual variability in hepatic DNL, assessed using the $\mathrm{D}_{2} \mathrm{O}$ technique in individuals with obesity with normal IHTG content and in those with obesity and NAFLD, and reported by others previously (14), and on the basis of whole-body insulin sensitivity, assessed as the glucose Rd during a HECP we reported previously (61), we estimated that 15 to 25 subjects per group would be needed to detect between-groups differences in hepatic DNL of $7.5 \%$ to $10 \%$ and between-groups differences in glucose $\mathrm{Rd}$ of 11 to $15 \mu \mathrm{mol} / \mathrm{kg} \mathrm{FFM} / \mathrm{min}$ using a 2 -sided test with a $\beta$ value of 0.9 and an $\alpha$ value of 0.05 . On the basis of the values for hepatic DNL measured in the obese-NAFLD group, we estimated that 6 subjects would be needed to detect a weight loss-induced $14 \%$ decrease in hepatic DNL with a $\beta$ value of 0.90 and an $\alpha$ value of 0.05 . These computations were performed using $\mathrm{G}^{*}$ Power 3.1.9.2 (62).

Study approval. All study subjects provided written, informed consent before participating in this study, which was approved by the Human Research Protection Office at the Washington University School of Medicine.

\section{Author contributions}

GIS, MKH, and SK designed the study. MS, ALO, EN, TF, and BWP performed sample analyses. GIS, GGS, MY, MC, and JWB conducted the clinical studies. CBS assessed IHTG content. GIS, $\mathrm{MKH}, \mathrm{ST}$, and SK interpreted the data and wrote the manuscript. All authors critically reviewed and edited the manuscript.

\section{Acknowledgments}

The authors thank Jennifer Shew and Frieda Custodio for their technical assistance; Kyleigh Kirbach, Janet Winkelmann, Sally Torbitzky, and the nurses of the Clinical and Translational Research Unit for their assistance in conducting the studies; and the study subjects for their participation. This study was supported by NIH grants DK56341 (Nutrition Obesity Research Center), DK20579 (Diabetes Research Center), DK52574 (Digestive Disease Research Center), and RR024992 (Clinical and Translational Science Award), and by grants from the Academy of Nutrition and Dietetics Foundation, the College of Natural Resources (UCB), and the Pershing Square Foundation.

Address correspondence to: Samuel Klein, Center for Human Nutrition, Washington University School of Medicine, 660 South Euclid Avenue, Campus Box 8031, St. Louis, Missouri 63110, USA. Phone: 314.362.8708; Fax: 314.362.8230; Email: sklein@wustl. edu. Or to: Marc Hellerstein, Department of Nutritional Sciences and Toxicology, 309 Morgan Hall, University of California at Berkeley, Berkeley, California 94720, USA. Phone: 510.64.0646; Fax: 510.642. 0535; E-mail: march@berkeley.edu.

CM's present address is: Department of Nutrition, University of California Davis, Davis, California, USA.
1. Korenblat KM, Fabbrini E, Mohammed BS, Klein S. Liver, muscle, and adipose tissue insulin action is directly related to intrahepatic triglyceride content in obese subjects. Gastroenterology. 2008;134(5):1369-1375.

2. Fabbrini E, et al. Intrahepatic fat, not visceral fat, is linked with metabolic complications of obesity. Proc Natl Acad Sci USA. 2009;106(36):15430-15435.

3. Speliotes EK, et al. Fatty liver is associated with dyslipidemia and dysglycemia independent of visceral fat: the Framingham Heart Study. Hepatology. 2010;51(6):1979-1987.

4. Hamaguchi M, et al. The metabolic syndrome as a predictor of nonalcoholic fatty liver disease. Ann Intern Med. 2005;143(10):722-728.

5. DeFilippis AP, et al. Nonalcoholic fatty liver disease and serum lipoproteins: the Multi-Ethnic Study of Atherosclerosis. Atherosclerosis. 2013;227(2):429-436.

6. Targher G, Byrne CD, Lonardo A, Zoppini G, Barbui C. Non-alcoholic fatty liver disease and risk of incident cardiovascular disease: A meta-analysis. J Hepatol. 2016;65(3):589-600.

7. Fabbrini E, Mohammed BS, Magkos F, Korenblat KM, Patterson BW, Klein S. Alterations in adipose tissue and hepatic lipid kinetics in obese men and women with nonalcoholic fatty liver disease. Gastroenterology. 2008;134(2):424-431.

8. Adiels M, et al. Overproduction of VLDL1 driven by hyperglycemia is a dominant feature of diabetic dyslipidemia. Arterioscler Thromb Vasc Biol. 2005;25(8):1697-1703.

9. Bugianesi E, et al. Insulin resistance in non- diabetic patients with non-alcoholic fatty liver disease: sites and mechanisms. Diabetologia. 2005;48(4):634-642.

10. Chalasani N, et al. Hepatic cytochrome P450 2E1 activity in nondiabetic patients with nonalcoholic steatohepatitis. Hepatology. 2003;37(3):544-550.

11. Sanyal AJ, et al. Nonalcoholic steatohepatitis: association of insulin resistance and mitochondrial abnormalities. Gastroenterology. 2001;120(5):1183-1192.

12. Donnelly KL, Smith CI, Schwarzenberg SJ, Jessurun J, Boldt MD, Parks EJ. Sources of fatty acids stored in liver and secreted via lipoproteins in patients with nonalcoholic fatty liver disease. J Clin Invest. 2005;115(5):1343-1351.

13. Fabbrini E, Sullivan S, Klein S. Obesity and nonalcoholic fatty liver disease: biochemical, 
metabolic, and clinical implications. Hepatology. 2010;51(2):679-689.

14. Lambert JE, Ramos-Roman MA, Browning JD, Parks EJ. Increased de novo lipogenesis is a distinct characteristic of individuals with nonalcoholic fatty liver disease. Gastroenterology. 2014;146(3):726-735.

15. Diraison F, Moulin P, Beylot M. Contribution of hepatic de novo lipogenesis and reesterification of plasma non esterified fatty acids to plasma triglyceride synthesis during non-alcoholic fatty liver disease. Diabetes Metab. 2003;29(5):478-485.

16. Hodson L, et al. Docosahexaenoic acid enrichment in NAFLD is associated with improvements in hepatic metabolism and hepatic insulin sensitivity: a pilot study. Eur J Clin Nutr. 2017;71(8):973-979.

17. Hellerstein MK, et al. Measurement of de novo hepatic lipogenesis in humans using stable isotopes. JClin Invest. 1991;87(5):1841-1852.

18. Diraison F, Yankah V, Letexier D, Dusserre E, Jones $\mathrm{P}$, Beylot M. Differences in the regulation of adipose tissue and liver lipogenesis by carbohydrates in humans. JLipid Res. 2003;44(4):846-853.

19. Marques-Lopes I, Ansorena D, Astiasaran I, Forga L, Martínez JA. Postprandial de novo lipogenesis and metabolic changes induced by a high-carbohydrate, low-fat meal in lean and overweight men. Am J Clin Nutr. 2001;73(2):253-261.

20. Faix D, et al. Quantification of menstrual and diurnal periodicities in rates of cholesterol and fat synthesis in humans. JLipid Res. 1993;34(12):2063-2075.

21. Timlin MT, Parks EJ. Temporal pattern of de novo lipogenesis in the postprandial state in healthy men. Am J Clin Nutr. 2005;81(1):35-42.

22. Lee JJ, et al. Palmitoleic acid is elevated in fatty liver disease and reflects hepatic lipogenesis. Am JClin Nutr. 2015;101(1):34-43.

23. Diraison F, Pachiaudi C, Beylot M. Measuring lipogenesis and cholesterol synthesis in humans with deuterated water: use of simple gas chromatographic/mass spectrometric techniques. J Mass Spectrom. 1997;32(1):81-86.

24. Lawitz EJ, et al. Acetyl-CoA carboxylase inhibitor GS-0976 for 12 weeks reduces hepatic de novo lipogenesis and steatosis in patients with nonalcoholic steatohepatitis. Clin Gastroenterol Hepatol. 2018;16(12):1983-1991.e3.

25. Shimomura I, Bashmakov Y, Ikemoto S, Horton JD, Brown MS, Goldstein JL. Insulin selectively increases SREBP-1c mRNA in the livers of rats with streptozotocin-induced diabetes. Proc Natl Acad Sci USA. 1999;96(24):13656-13661.

26. Browning JD, Horton JD. Molecular mediators of hepatic steatosis and liver injury. JClin Invest. 2004;114(2):147-152.

27. Yamashita H, et al. A glucose-responsive transcription factor that regulates carbohydrate metabolism in the liver. Proc Natl Acad Sci USA. 2001;98(16):9116-9121.

28. Stoeckman AK, Towle HC. The role of SREBP-1c in nutritional regulation of lipogenic enzyme gene expression.J Biol Chem. 2002;277(30):27029-27035.

29. Koo SH, Dutcher AK, Towle HC. Glucose and insulin function through two distinct transcription factors to stimulate expression of lipogenic enzyme genes in liver. J Biol Chem.
2001;276(12):9437-9445.

30. Foretz M, Guichard C, Ferré P, Foufelle F. Sterol regulatory element binding protein-1c is a major mediator of insulin action on the hepatic expression of glucokinase and lipogenesis-related genes. Proc Natl Acad Sci USA. 1999;96(22):12737-12742.

31. Brown MS, Goldstein JL. Selective versus total insulin resistance: a pathogenic paradox. Cell Metab. 2008;7(2):95-96.

32. Shimomura I, Bashmakov Y, Horton JD. Increased levels of nuclear SREBP-1c associated with fatty livers in two mouse models of diabetes mellitus. J Biol Chem. 1999;274(42):30028-30032.

33. Shimomura I, Matsuda M, Hammer RE, Bashmakov Y, Brown MS, Goldstein JL. Decreased IRS-2 and increased SREBP-1c lead to mixed insulin resistance and sensitivity in livers of lipodystrophic and ob/ob mice. Mol Cell. 2000;6(1):77-86.

34. Li S, Brown MS, Goldstein JL. Bifurcation of insulin signaling pathway in rat liver: mTORC1 required for stimulation of lipogenesis, but not inhibition of gluconeogenesis. Proc Natl Acad Sci USA. 2010;107(8):3441-3446.

35. Ruge $\mathrm{T}$, et al. Fasted to fed trafficking of fatty acids in human adipose tissue reveals a novel regulatory step for enhanced fat storage. J Clin Endocrinol Metab. 2009;94(5):1781-1788.

36. Angulo P. Nonalcoholic fatty liver disease. $N$ Engl JMed. 2002;346(16):1221-1231.

37. Rosqvist F, et al. Fasting hepatic de novo lipogenesis is not reliably assessed using circulating fatty acid markers. Am JClin Nutr. 2019;109(2):260-268.

38. Vedala A, Wang W, Neese RA, Christiansen MP, Hellerstein MK. Delayed secretory pathway contributions to VLDL-triglycerides from plasma NEFA, diet, and de novo lipogenesis in humans. J Lipid Res. 2006;47(11):2562-2574.

39. Shimano H, Horton JD, Shimomura I, Hammer RE, Brown MS, Goldstein JL. Isoform 1c of sterol regulatory element binding protein is less active than isoform 1a in livers of transgenic mice and in cultured cells. JClin Invest. 1997;99(5):846-854.

40. Lima-Cabello E, et al. Enhanced expression of pro-inflammatory mediators and liver X-receptor-regulated lipogenic genes in non-alcoholic fatty liver disease and hepatitis C. Clin Sci. 2011;120(6):239-250.

41. Higuchi N, et al. Liver X receptor in cooperation with SREBP-1c is a major lipid synthesis regulator in nonalcoholic fatty liver disease. Hepatol Res. 2008;38(11):1122-1129.

42. Mitsuyoshi H, et al. Analysis of hepatic genes involved in the metabolism of fatty acids and iron in nonalcoholic fatty liver disease. Hepatol Res. 2009;39(4):366-373.

43. Kohjima M, et al. Re-evaluation of fatty acid metabolism-related gene expression in nonalcoholic fatty liver disease. Int $\mathrm{J} \mathrm{Mol} \mathrm{Med.}$ 2007;20(3):351-358.

44. Benhamed F, et al. The lipogenic transcription factor ChREBP dissociates hepatic steatosis from insulin resistance in mice and humans. JClin Invest. 2012;122(6):2176-2194.

45. Choi CS, et al. Suppression of diacylglycerol acyltransferase-2 (DGAT2), but not DGAT1, with antisense oligonucleotides reverses diet-induced hepatic steatosis and insulin resistance. J Biol
Chem. 2007;282(31):22678-22688.

46. Xia JY, et al. Targeted induction of ceramide degradation leads to improved systemic metabolism and reduced hepatic steatosis. Cell Metab. 2015;22(2):266-278.

47. Kim CW, et al. Acetyl CoA carboxylase inhibition reduces hepatic steatosis but elevates plasma triglycerides in mice and humans: a bedside to bench investigation. Cell Metab. 2017;26(2):394-406.e6.

48. Buse JB, et al. Randomized clinical trial comparing basal insulin peglispro and insulin glargine in patients with type 2 diabetes previously treated with basal insulin: IMAGINE 5. Diabetes Care. 2016;39(1):92-100.

49. Henry RR, et al. Basal insulin peglispro demonstrates preferential hepatic versus peripheral action relative to insulin glargine in healthy subjects. Diabetes Care. 2014;37(9):2609-2615.

50. Kirk E, et al. Dietary fat and carbohydrates differentially alter insulin sensitivity during caloric restriction. Gastroenterology. 2009;136(5):1552-1560.

51. Magkos F, et al. Effects of moderate and subsequent progressive weight loss on metabolic func tion and adipose tissue biology in humans with obesity. Cell Metab. 2016;23(4):591-601.

52. Mittendorfer B, Magkos F, Fabbrini E, Mohammed BS, Klein S. Relationship between body fat mass and free fatty acid kinetics in men and women. Obesity (Silver Spring). 2009;17(10):1872-1877.

53. Le TA, et al. Effect of colesevelam on liver fat quantified by magnetic resonance in nonalcoholic steatohepatitis: a randomized controlled trial. Hepatology. 2012;56(3):922-932.

54. Mifflin MD, St Jeor ST, Hill LA, Scott BJ, Daugherty SA, Koh YO. A new predictive equation for resting energy expenditure in healthy individuals. Am J Clin Nutr. 1990;51(2):241-247.

55. Klein S, et al. Absence of an effect of liposuction on insulin action and risk factors for coronary heart disease. NEngl JMed. 2004;350(25):2549-2557.

56. Friedewald WT, Levy RI, Fredrickson DS. Estimation of the concentration of low-density lipoprotein cholesterol in plasma, without use of the preparative ultracentrifuge. Clin Chem. 1972;18(6):499-502.

57. Mittendorfer B, Horowitz JF, Klein S. Gender differences in lipid and glucose kinetics during short-term fasting. Am J Physiol Endocrinol Metab. 2001;281(6):E1333-E1339.

58. Strawford A, Antelo F, Christiansen M, Hellerstein MK. Adipose tissue triglyceride turnover, de novo lipogenesis, and cell proliferation in humans measured with 2H2O. Am J Physiol Endocrinol Metab. 2004;286(4):E577-E588.

59. Allison DB, Paultre F, Maggio C, Mezzitis N, Pi-Sunyer FX. The use of areas under curves in diabetes research. Diabetes Care. 1995;18(2):245-250.

60. Magkos F, Patterson BW, Mohammed BS, Klein S, Mittendorfer B. Women produce fewer but triglyceride-richer very low-density lipoproteins than men.J Clin Endocrinol Metab. 2007;92(4):1311-1318.

61. Fabbrini E, et al. Metabolically normal obese people are protected from adverse effects following weight gain. JClin Invest. 2015;125(2):787-795.

62. Faul F, Erdfelder E, Lang AG, Buchner A. G*Power 3: a flexible statistical power analysis program for the social, behavioral, and biomedical sciences. Behav Res Methods. 2007;39(2):175-191. 